\title{
Pharmacological inhibition of Rho-kinase (ROCK) signaling enhances cisplatin resistance in neuroblastoma cells
}

\author{
CATHARINE A. STREET, ALISSA A. ROUTHIER, CARRIE SPENCER, ASHLEY L. PERKINS, \\ KATHERINE MASTERJOHN, ALEXANDER HACKATHORN, JOHN MONTALVO, \\ EMILY A. DENNSTEDT and BRAD A. BRYAN
}

Ghosh Science and Technology Center, Worcester State College, Worcester, MA, USA

Received June 24, 2010; Accepted July 30, 2010

DOI: 10.3892/ijo_00000781

\begin{abstract}
The role of the RhoA/Rho kinase (ROCK) signaling pathway in cell survival remains a very controversial issue, with its activation being pro-apoptotic in many cell types and anti-apoptotic in others. To test if ROCK inhibition contributes to tumor cell survival or death following chemotherapy, we treated cisplatin damaged neuroblastoma cells with a pharmacological ROCK inhibitor (Y27632) or sham, and monitored cell survival, accumulation of a chemoresistant phenotype, and in vivo tumor formation. Additionally, we assayed if ROCK inhibition altered the expression of genes known to be involved in cisplatin resistance. Our studies indicate that ROCK inhibition results in increased cell survival, acquired chemoresistance, and enhanced tumor survival following cisplatin cytotoxicity, due in part to altered expression of cisplatin resistance genes. These findings suggest that ROCK inhibition in combination with cisplatin chemotherapy may lead to enhanced tumor chemoresistance in neuroblastoma.
\end{abstract}

\section{Introduction}

Neoplastic resistance is one of the major obstacles preventing the successful chemotherapeutic treatment of tumors. While the incidence of anti-cancer drug resistance varies greatly between tumor types, one clinical observation remains consistent, once a tumor displays properties of chemotherapy resistance, patient survival rates are considerably diminished. Cis-diamminedichloro-platinum (cisplatin) and its derivatives have become some of the most important therapies in the clinical management of tumors over the last several decades, acting as cytotoxic agents by forming covalent platinum adducts with DNA. These adducts are subsequently detected by the cell's DNA damage response machinery, leading to cell

Correspondence to: Dr Brad A. Bryan, Ghosh Science and Technology Center, ST310D, Worcester State College, 486 Chandler Street, Worcester, MA 01602-2597, USA

E-mail: brad.bryan@worcester.edu

Key words: Rho-kinase, ROCK, RhoA, cisplatin, neuroblastoma cycle arrest, or, if the DNA damage is not sufficiently repaired, activation of the apoptotic cascade. Cisplatin demonstrates good clinical efficiency in patients whose tumors possess functional apoptotic pathways; however relatively lower efficiency is observed in those with drug-resistant tumors where the major apoptotic pathways are disrupted. While the hallmark of classical tumor drug resistance is often deregulation of cell survival/apoptotic pathways, cisplatin-resistance can be gained through several other mechanisms including reduced drug uptake, enhanced drug efflux, increased metabolic drug inactivation, and enhanced adduct repair.

Numerous reports have implicated deregulation of the Rho-GTPase family of proteins in cancer, and because of their essential functions in regulating the cytoskeleton, Rho-GTPase proteins and their effectors are promising and actively researched targets for the development of novel anti-cancer drugs. Rho-proteins are significantly elevated in a variety of tumors (1-5), and are reported to strongly contribute to the metastatic behavior of some cancers $(6,7)$. For instance, studies utilizing in vitro models have suggested that RhoA and its downstream effector Rho-kinase (ROCK) regulate migration, invasion, proliferation, and survival of cancer cell lines. Moreover, ROCK inhibition has been shown to block invasion of prostate tumors (8), increase apoptosis in glioma tumors (9), inhibit melanoma tumor growth (10), and, in combination with Cidofovir, block metastasis of human papilloma virus positive tumor cells to the lung (11). Conflicting reports have addressed the role of ROCK proteins in cell survival, with activation of ROCK being pro- or antiapoptotic in a cell-type dependent manner (12). Interestingly, caspase cleavage of ROCK leads to its constitutive activation, and this process is reportedly necessary for apoptosis (13). Furthermore, ROCK activity has been shown to drive apoptotic membrane blebbing, cell fragmentation, and phagocytosis of apoptotic bodies $(14,15)$. In contrast, ROCK reportedly phosphorylates phosphatase and tensin homologue (PTEN) and induces its negative regulation of the pro-survival phosphoinositol-3-kinase (PI3K) pathway (16). The mechanism balancing this dual role of ROCK proteins in regulating survival versus apoptosis is largely unknown, and given that ROCK inhibitors are of interest clinically for tumor treatment, we sought to determine if ROCK signaling is involved in mediating chemotherapeutic resistance. In this study we demonstrate that pharmacological inhibition of ROCK with 
the small molecule inhibitor Y27632 leads to increased survival of human neuroblastoma cells and tumors following cytotoxic chemotherapeutic treatment. Our data demonstrate that a combination of increased cell proliferation and alteration in the expression of genes previously established to be involved in cisplatin resistance in tumors account for the chemoresistant phenotype observed in ROCK inhibited cells.

\section{Materials and methods}

Cell lines and treatments. SK-N-SH, SHEP, and NGP human neuroblastoma cells (a generous gift from Dr Rani George, Harvard Medical School) were cultured in DMEM supplemented with $10 \%$ fetal bovine serum, $100 \mathrm{U}$ penicillin, and $100 \mu \mathrm{g}$ streptomycin. The working concentrations of chemicals used in this study are as follows: Y27632 $(10 \mu \mathrm{M})$, cisplatin $(10 \mu \mathrm{M})$, paclitaxel $(5 \mu \mathrm{M})$, busulfan $(10 \mu \mathrm{M}), 3 \%$ hydrogen peroxide (diluted 1:10,000), $\alpha$-pifithrin $(30 \mu \mathrm{M})$, verapamil $(20 \mu \mathrm{M})$, and NG123 $(10 \mu \mathrm{M})$. Cells were treated with ultraviolet-C (UV) at $50 \mathrm{~J} / \mathrm{m}^{2}$. Cisplatin, paclitaxel, busulfan, and hydrogen peroxide were added to the cells for $24 \mathrm{~h}$ and then removed via washing. Y27632, $\alpha$-pifithrin, verapamil, and NG123 were maintained throughout the length of the experiment. The double thymidine block to arrest cells at the G1/S transition was performed as previously reported (17).

Analysis of data. All assays were performed in triplicate and repeated via independent experiments. Data are presented as the average plus or minus the standard deviation. Statistical significance was set at $\mathrm{p}<0.05$, determined by Student's t-test.

Fluorescence activated cell sorting (FACs). Cells were trypsinized, washed in PBS, and fixed overnight at $4^{\circ} \mathrm{C}$ in $70 \%$ ethanol in PBS. Cells were washed two times in PBS and resuspended in PBS with $50 \mu \mathrm{g} / \mathrm{ml}$ propidium iodide and $50 \mu \mathrm{g} / \mathrm{ml}$ RNase A. Cells were incubated at $4^{\circ} \mathrm{C}$ overnight and analyzed using FACs.

Semi-quantitative reverse transcriptase polymerase chain reaction $(R T-P C R)$. RNA was extracted using TRIzol reagent (Invitrogen) according to the manufacturer's instructions. RNA was converted to cDNA using Verso cDNA kit (Thermo Scientific) according to the manufacturer's instructions. Oligo dT's were utilized in the cDNA conversion for all mRNAs except histones, for which random hexamers were used. PCR amplification of specific cDNAs was performed using primers designed by Primer Blast. GAPDH was always used as a control.

Proliferation and survival assays. For proliferation and survival assays, manual cell counts and indirect measurements using 3-(4,5-Dimethylthiazol-2-yl)-2,5-diphenyltetrazolium bromide (MTT) assays (Cayman Chemical) were performed. MTT assays were performed according to the manufacturer's instructions.

Single cell gel electrophoresis assay. Single cell gel electrophoresis assay was performed as previously reported (18). Hydrogen peroxide (3\%) of cells for 30 min prior to harvesting was used as a positive control.
Glutathione-S-transferase (GST) assay. The conjugation of 1-chloro-2,4-dinitrobenzene (CDNB) with reduced glutathione was measured at $340 \mathrm{~nm}$ as previously reported (19).

Chorioallantoic membrane (CAM) assay. CAM assays were performed as previously described (20). NGP neuroblastoma cells $(100,000)$ per gelatin implant were utilized since SK$\mathrm{N}-\mathrm{SM}$ cells failed to form tumors in the CAM assay. Every two days, $100 \mu \mathrm{l}$ of sham or $100 \mu \mathrm{l}$ of a $10 \mu \mathrm{M}$ solution of Y27632 was added to the implanted sponge.

\section{Results}

ROCK inhibition protects human neuroblastoma cells from cytotoxic cell death. The ROCK signaling pathway has previously been reported to enhance cell survival in some cell types, while increasing apoptosis in others. The mechanism behind this apparent contradiction is largely unknown. To determine what role ROCK signaling plays in neuroblastoma cell survival, we treated sham or Y27632 treated SK-N-SM human neuroblastoma cells with cisplatin, paclitaxel, busulfan, hydrogen peroxide, or ultraviolet light and measured cell survival $72 \mathrm{~h}$ post treatment. As indicated in Fig. 1, pharmacological ROCK inhibition led to significantly increased cell survival in all treatments.

To determine if this trend is replicated in other neuroblastoma cell lines, we challenged SK-N-SM, SHEP, and NGP human neuroblastoma cell lines with cisplatin treatment plus the presence or absence of Y27632, and quantified cell survival in each condition after 5 days. Y27632 treatment resulted in a significant increase in the number of remaining cells compared to the control for all neuroblastoma cell lines tested (Fig. 2A and B, first treatment). We then pooled the surviving cells from each treatment for further culture, and, upon replenishment of the cell population, these cells were subjected to subsequent rounds of $10 \mu \mathrm{M}$ cisplatin to select for cisplatin-resistant neuroblastoma lines. By the second round of cisplatin treatment the large majority of the Y27632 treated cells from all cell lines remained viable following treatment, compared to $<30 \%$ of the control cells (Fig. 2A and $\mathrm{B}$, second treatment). Though a portion of the control cells survived the second round of cisplatin treatment, these surviving cells failed to undergo proliferation and recovery even after 3 weeks of culture. In contrast, following the second round of cisplatin treatment, a significant population of the Y27632 treated cells began to proliferate within one week, suggesting Y27632 shortened the recovery time after the cytotoxic insult. By the third round of $10 \mu \mathrm{M}$ cisplatin treatment, Y27632 treated cells appeared unaffected by cisplatin injury and remained largely in a proliferative state (data not shown).

Cisplatin treated sham and Y27632 treated neuroblastoma cells were collected $48 \mathrm{~h}$ post-treatment for cell cycle analysis using flow cytometry. As indicated in Fig. 2C (data shown only for SK-N-SM cells, similar results in the other cell lines), cisplatin treatment resulted in a substantial sub-G1 apoptotic population which was ablated by Y27632 treatment. Interestingly, cisplatin treated Y27632 cells demonstrated a markedly different pattern of cell cycle progression compared to cisplatin treatment alone. Collectively, these data indicate 


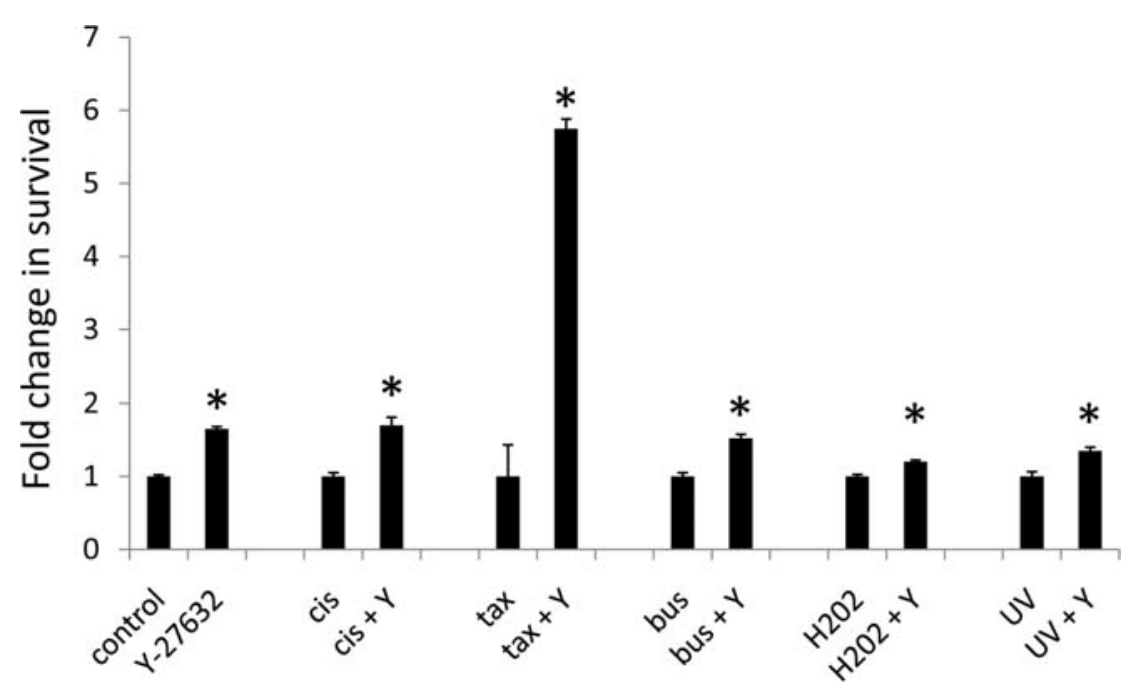

Figure 1. ROCK inhibition leads to enhanced survival of neuroblastoma cells following cytotoxic treatment. SK-N-SH neuroblastoma cells were treated with $10 \mu \mathrm{M}$ cisplatin (cis), $5 \mu \mathrm{M}$ paclitaxel (tax), $10 \mu \mathrm{M}$ busulfan (bus), 1:10,000 dilution of $3 \%$ hydrogen peroxide $\left(\mathrm{H}_{2} \mathrm{O}_{2}\right.$ ), or $50 \mathrm{~J} / \mathrm{m}^{2}$ ultraviolet light (UV) plus the presence (Y) or absence of Y27632. The number of surviving cells was measured after $72 \mathrm{~h}$. Data are displayed as the fold change in survival compared to the control.
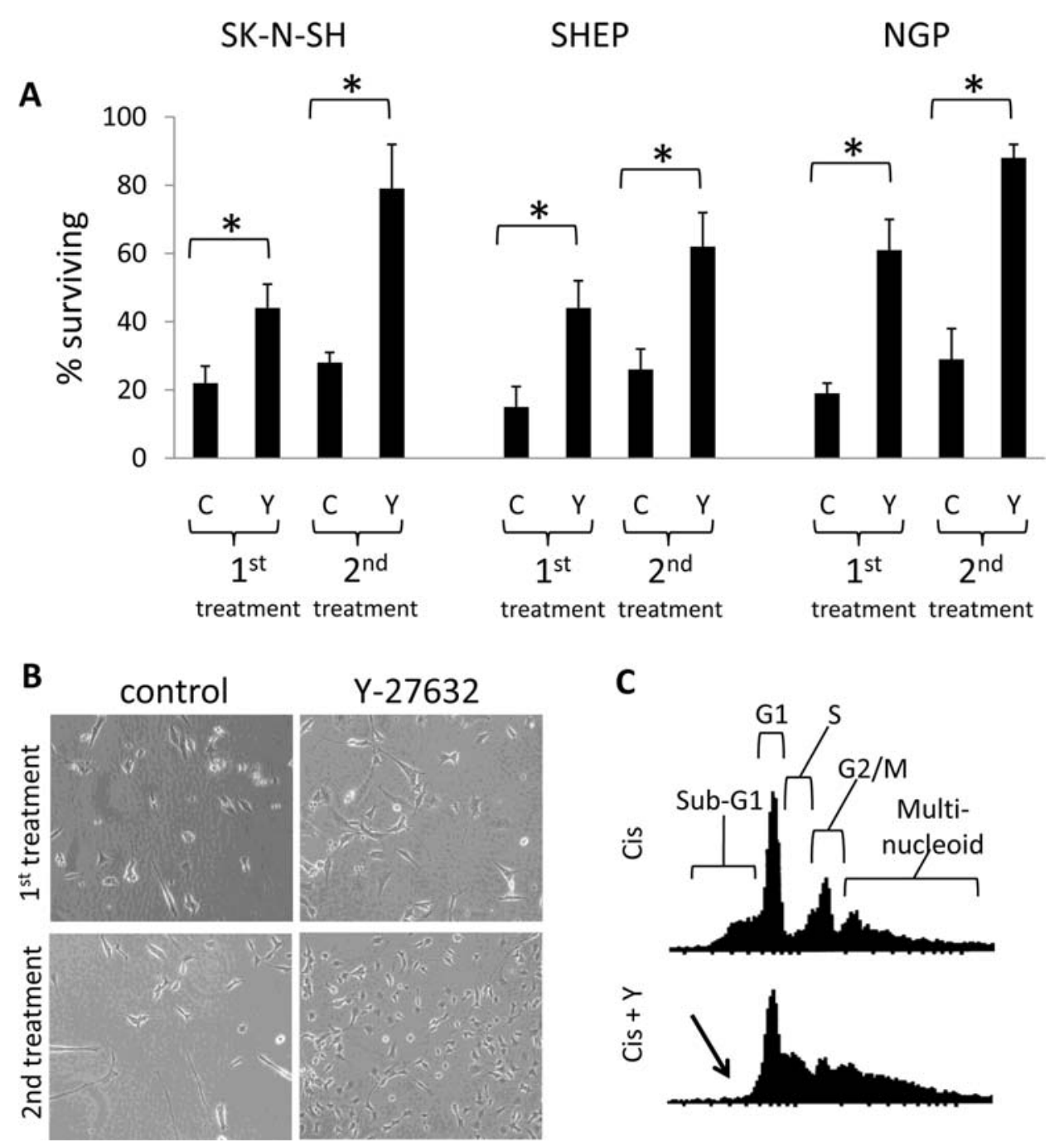

Figure 2. Y27632 treatment leads to survival and chemoresistance in cisplatin-treated neuroblastoma cells. (A) SK-N-SH (photographed in B), SHEP, and NGP human neuroblastoma cells were treated with $10 \mu \mathrm{M}$ cisplatin plus the presence $(\mathrm{Y})$ or absence $(\mathrm{C})$ of $\mathrm{Y} 27632$ and the number of surviving cells was measured by MTT assay after five days (1st treatment). The surviving cells from the first treatment were then pooled and expanded. These cells were then subjected to a second round of $10 \mu \mathrm{M}$ cisplatin, and the number of surviving cells was measured by MTT assay after 5 days (2nd treatment). (B) Representative images of SK-N-SM cells 5 days after treatment from (A). (C) Flow cytometric cell cycle analysis of propidium iodide stained SK-N-SH cells collected $48 \mathrm{~h}$ following $10 \mathrm{mM}$ cisplatin plus the presence (Cis $+\mathrm{Y}$ ) or absence (Cis) of Y27632. Locations of the cell cycle phases are diagrammed in the upper panel. The arrow indicates the lack of a sub-G1 population in the Y27632 treated cells. 
A

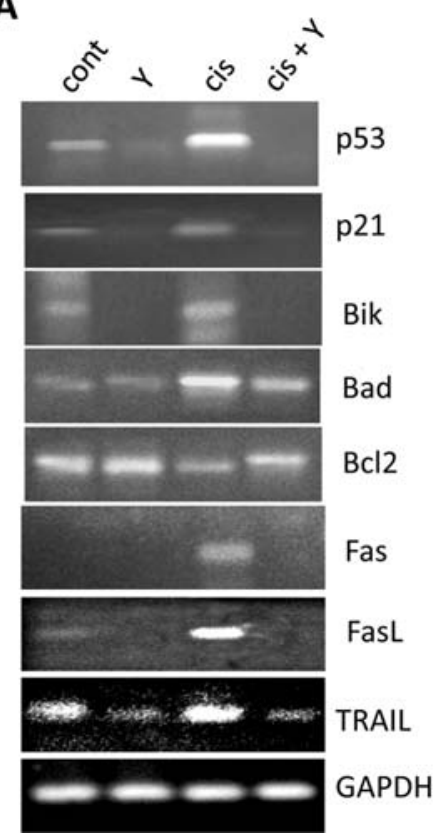

B
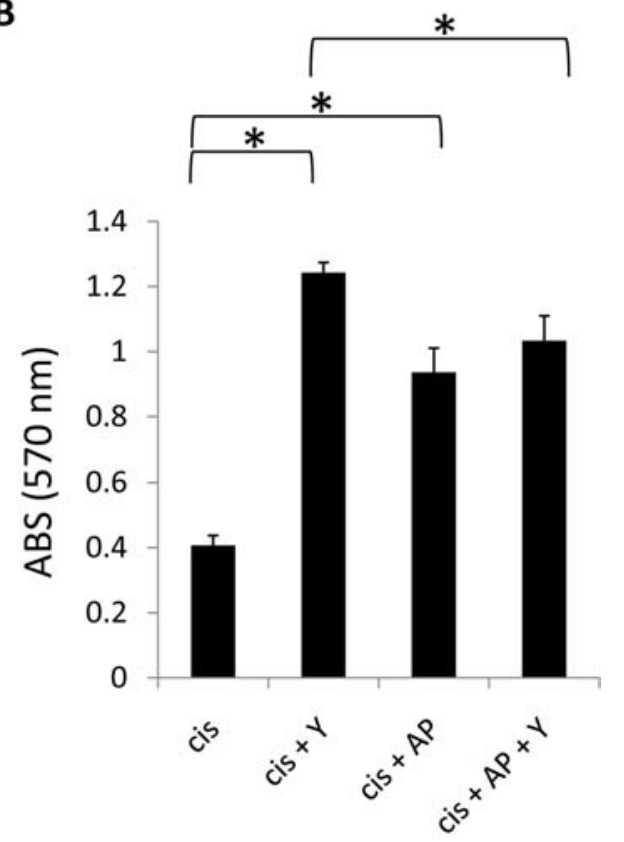

Figure 3. Y27632 treatment of neuroblastoma cells alters the expression of pro- and anti-apoptotic genes. (A) Semi-quantitative RT-PCR analysis measuring steady state mRNA expression of DNA damage response genes (p53 and p21), intrinsic apoptotic regulators (Bik, Bad, and Bcl2), and extrinsic apoptotic regulators (Fas, FasL, and Trail) in control and Y27632 treated SK-N-SH cells which were incubated for 24 h following the indicated treatment. (B) SK-N-SM cells were treated with indicated combinations of cisplatin (cis), Y27632 (Y), or the pharmacological p53 inhibitor $\alpha$-pifithrin (AP), and survival was measured after 5 days.

that ROCK signaling in neuroblastoma cell lines is proapoptotic, and pharmacological inhibition of this pathway increases cell survival and drug resistance following cytotoxic insult.

Rho-kinase inhibition alters expression of intrinsic and extrinsic apoptotic regulators in SK-N-SM cells. To determine the molecular mechanism by which the Rho/ROCK pathway modulates neuroblastoma cell death in response to cisplatin, we focused our efforts on SK-N-SH cells. The steady state mRNA expression levels of DNA damage response genes (p53 and p21), intrinsic apoptotic regulators (Bik, Bad, and $\mathrm{Bcl} 2$ ), and extrinsic apoptotic regulators (Fas, Fas ligand (FasL), and Trail) were analyzed using semi-quantitative RT-PCR in control and Y27632 treated SK-N-SH cells which were incubated for $24 \mathrm{~h}$ with either sham or cisplatin. Compared to control cells in both the sham and cisplatin treatments, pharmacological ROCK inhibition resulted in down-regulation of the steady state mRNA levels of the cell cycle inhibitors p53 and p21, up-regulation of the prosurvival $\mathrm{Bcl} 2$, and down-regulation of the pro-apoptotic Bik, Bad, Fas, FasL, and Trail (Fig. 3A). These data suggest that ROCK modulation of apotosis impinges on altering the expression levels of both intrinsic and extrinsic apoptotic mediators.

p53 activation and its subsequent up-regulation of expression is an essential component of the intact DNA-damage response following cytotoxic treatment. To determine if p53 signaling is involved in ROCK-mediated modulation of apoptosis, we treated cisplatin or cisplatin + Y27632 treated SK-N-SH cells with the p53 inhibitor $\alpha$-pifithrin, which reversibly blocks p53-dependent transcriptional activation and apoptosis induction, and measured cell survival 5 days post-treatment. As expected, p53 inhibition via $\alpha$-pifithrin addition to cisplatin treated SK-N-SH cells significantly increased cell survival (Fig. 3B). Interestingly, p53 inhibition of cisplatin + Y27632 treated cells resulted in a small but significant decrease in survival compared to cisplatin + Y27632 treated cells with a functional p53 pathway. These data suggest that the increase in cell survival observed in Y27632 treated cells following cisplatin addition utilizes, but is not wholly dependent, on a functional p53 signaling pathway, and thus may partly employ initiation of the DNA damage response machinery to be fully effective.

ROCK inhibition enhances SK-N-SM cell survival following cisplatin treatment via promotion of cell proliferation, upregulation of DNA damage repair systems, and modulation of cisplatin uptake/efflux. Recent studies have explicitly defined alterations in cell cycle progression, expression/activity of nucleotide excision repair (NER) genes, activity of glutathioneS-transferase (GST), expression of copper transporter 1 (CTR1) (a copper transporter which serves as the cellular cisplatin import protein), and expression of a handful of ABC-binding cassette $(\mathrm{ABC})$ multidrug efflux pumps as being commonly mis-regulated during cisplatin resistance across a number of cell lines and tumor types (21).

Cell cycle analysis of control and Y27632 treated SK$\mathrm{N}-\mathrm{SM}$ cells indicated that pharmacological ROCK-inhibition markedly altered the pattern of cell cycle progression in both cisplatin treated (Fig. 2C) and proliferating cultures (Fig. 4A). Indeed, Y27632 treatment of SK-N-SM cells resulted in a significant increase in cell proliferation compared to control cells (Fig. 4B). To confirm that Y27632 treatment alters progression through the cell cycle phases, we arrested SK$\mathrm{N}-\mathrm{SM}$ cells at the G1/S transition using a double thymidine 


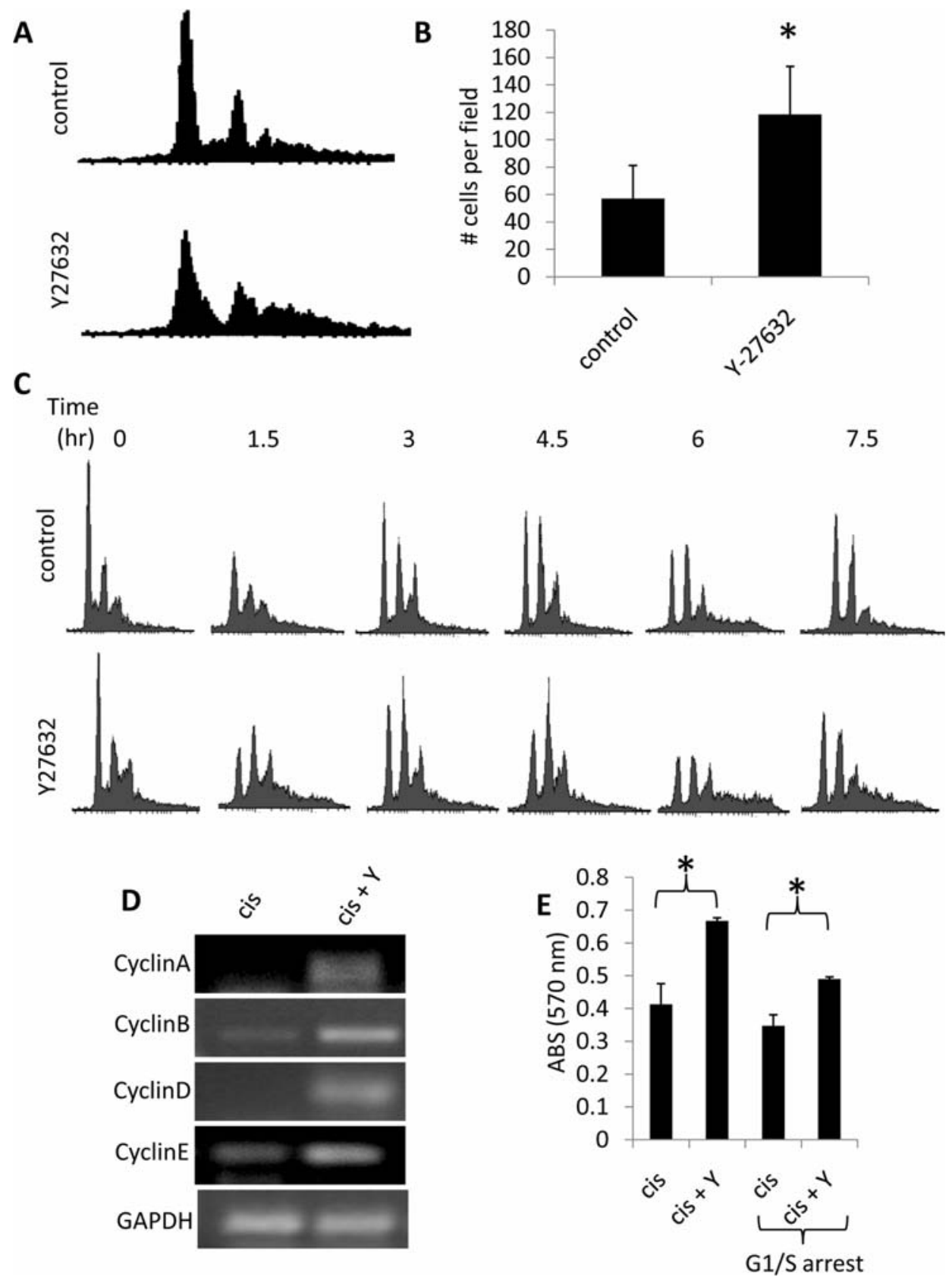

Figure 4. Enhanced cisplatin resistance of Y27632 treated SK-N-SM neuroblastoma cells is due in part to changes in cellular proliferation. (A) Flow cytometric cell cycle analysis of propidium iodide stained steady state SK-N-SH cells treated with sham or Y27632. (B) Proliferation assay of sham vs. Y27632 treated SK-N-SM cells. C. SK-N-SM cells were arrested at the G1/S transition with a double thymidine block. Cells were released from the block and samples were taken at the indicated time-points to analyze progression through the cell cycle using flow cytometric cell cycle analysis. (D) Semi-quantitative RT-PCR analysis measuring steady state mRNA expression of cycle cycle regulated genes in cisplatin (cis) vs. Y27632 (Y) treated SK-N-SM cells. (E) Survival of cisplatin treated SK-N-SM cells, either in steady state or arrested at the G1/S transition using a double thymidine block (G1/S arrest) was determined using an MTT assay 3 days post-treatment.

block. Cells were then treated with control or Y27632, released from the block to progress through the cell cycle, and collected at 1.5-h intervals for cell cycle analysis (Fig. 4C). We acknowledge that interpretation of the cell cycle plots from this experiment is compounded by the fact that this cell line contains a significant portion of multinucleated cells (as indicated by the numerous right shifted peaks present in Figs. 2C and 4A, and the lack of a single G1 peak in Fig. 4C due to this issue (4), therefore the G2/M peak is composed of single nucleated cells in $\mathrm{G} 2 / \mathrm{M}$ as well as double nucleated cells in G1. However, it is clear from the data that Y27632 treated cells progress through the cell cycle at a more rapid rate than control cells as evidenced by the swift reduction in the G1 peak by $1.5 \mathrm{~h}$ after the release in the Y27632 treated cells, with a similar cell cycle plot not seen until $6 \mathrm{~h}$ after the release in the control cells. Analysis of cyclin expression in cisplatin treated sham or Y27632 SK-N-SM cells demonstrated increased cyclin A, B, D, and E steady state mRNA expression levels in Y27632 treated cells (Fig. 4D), corroborating our proliferation data. As a control, no change in histone steady state mRNA expression was observed for either condition. To determine if the observed increase in proliferation rate and alteration in cell cycle progression in Y27632 treated cells (Fig. 4A-C) contributes to increased cisplatin resistance, 
A

A $3 \% \mathrm{H} 2 \mathrm{O} 2$

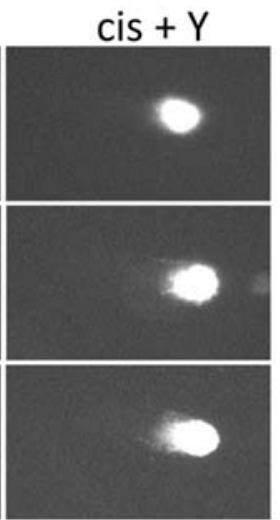

B

is $i^{x}$
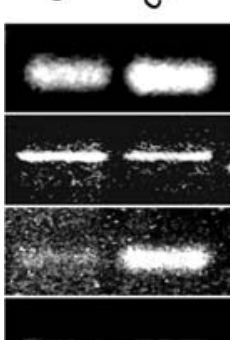

congever
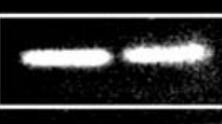

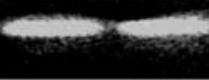

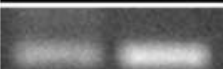

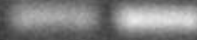

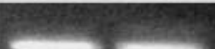

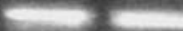

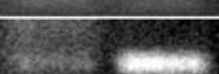

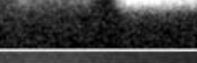

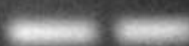

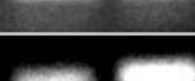

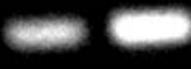

$=0$
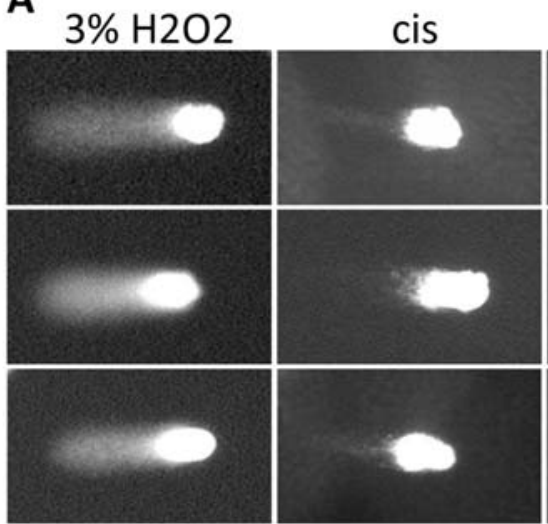

C

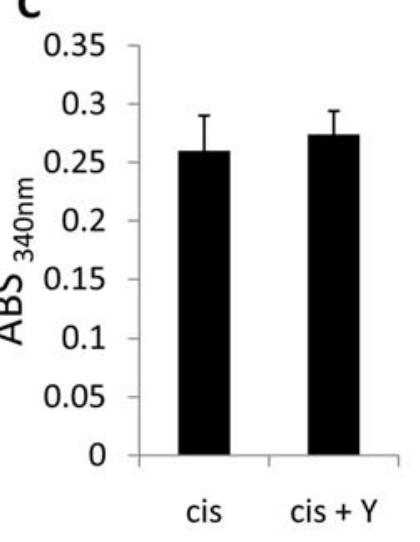

$\mathbf{E}$
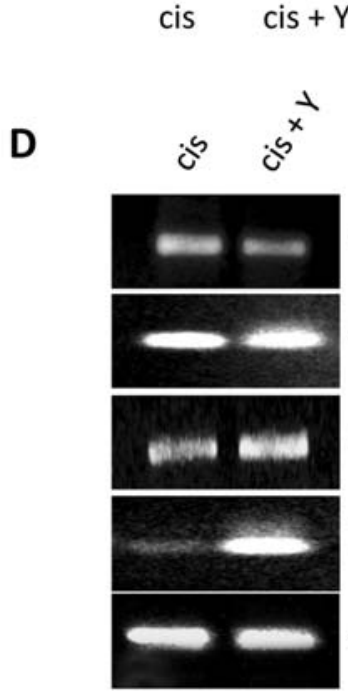

PCNA

Rad23

ERCC1

GAPDH

\section{CTR1}

ATP7A

ATP7B

MRP2

GAPDH

RPA
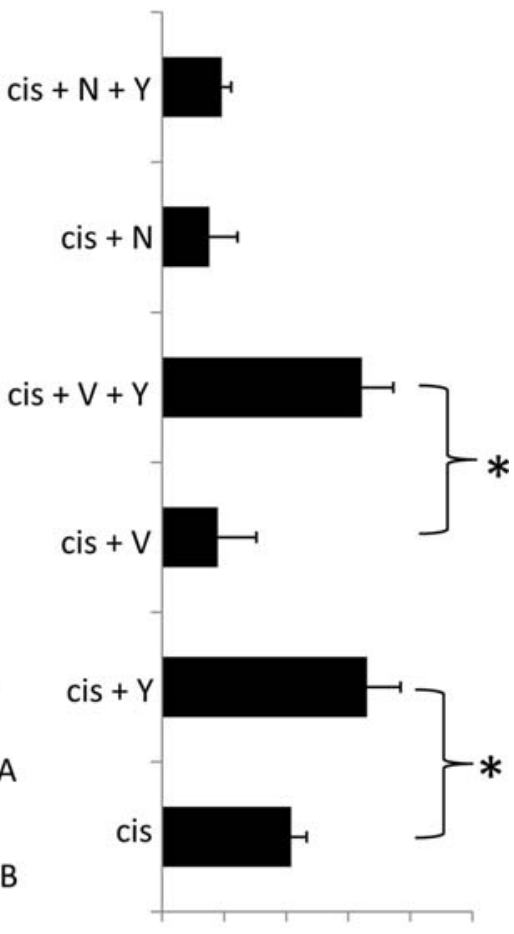

$\begin{array}{llllll}0 & 0.1 & 0.2 & 0.3 & 0.4 & 0.5\end{array}$

$\operatorname{ABS}(570 \mathrm{~nm})$

Figure 5. Y27632 treatment of SK-N-SM neuroblastoma cells induces alterations in the expression of known cisplatin resistance genes. (A) Single cell gel electrophoresis assay of cisplatin vs cisplatin + Y27632 treated SK-N-SM cells. $\mathrm{H}_{2} \mathrm{O}_{2}$ was used as a positive control. (B-D) RT-PCR expression analysis of DNA damage repair genes (B), glutathione-S-transferase activity (C), and RT-PCR expression analysis of cisplatin influx/efflux modulators (D) expressed in cisplatin vs cisplatin + Y-27632 treated SK-N-SM cells. (E) Survival of cispatin (cis) vs. Y-27632 (Y) treated SK-N-SM cells in the presence or absense of the cisplatin exflux inhibitor verapamil (V) or the DNA repair inhibitor $N G 123(\mathrm{~N})$.

we arrested SK-N-SM cells at the G1/S transition using a double thymidine block, treated the cells with cisplatin or cisplatin + Y27632, and subsequently measured survival. Y27632 + cisplatin treated cells which were arrested at the G1/S transition (and could not proliferate for the duration of the experiment) still demonstrated a survival advantage over cisplatin treatment alone, however this advantage was significantly less than the level observed in cisplatin + Y27632 treated cells that were freely progressing through the cell cycle (Fig. 4E). The data suggest that Y27632 treatment increases proliferation of SK-N-SM cells, and this increased proliferation rate partially contributes to the survival advantage of Y27632 treated cells following cisplatin insult.

To determine if ROCK inhibition leads to alterations in DNA damage repair, single cell gel electrophoresis assays measuring DNA breakage were performed on cisplatin and cisplatin + Y27632 treated SK-N-SM treated cells. As demonstrated in Fig. 5A, Y27632 treatment significantly shortened tail length of cisplatin treated cells compared to cisplatin treatment alone, suggesting that ROCK inhibition leads to 
A

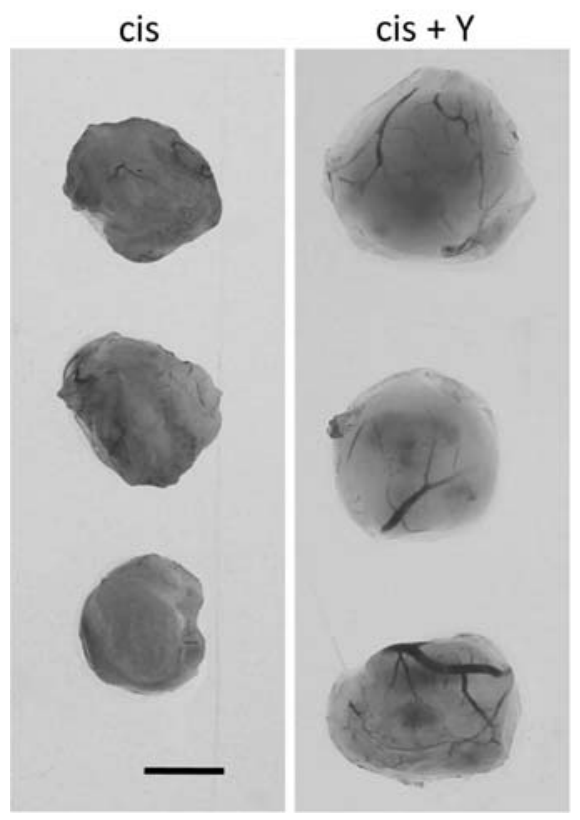

B

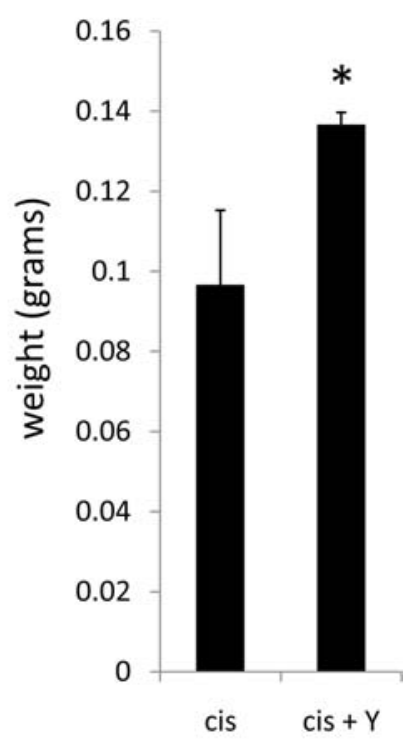

Figure 6. Y27632 treatment of NGP neuroblastoma tumors enhances CAM tumor growth. (A) NGP neuroblastoma CAM tumors were grown as indicated above. Cisplatin (cis) and cisplatin + Y27632 (cis + Y) treated tumors were harvested 5 days post-implantation. Scale bar equals 0.25 cm. (B) Quantification of tumor weight.

either a reduction in DNA damage or an increase in DNA damage repair. To determine the molecular mechanism behind this observation, we tested the effects of pharmacological ROCK inhibition on established targets involved in cisplatin resistance through expression or biochemical activity analysis of cisplatin or cisplatin + Y27632 treated SK-N-SH cells cultured for five days after the initial cisplatin insult. Our data indicate that Y27632 upregulates the expression of several NER genes (XPA, XPC, XPD, XPG, RPA, and ERCC1) and drug efflux pumps (ATPB2 and MRP2), while reducing expression of CTR1 (Fig. 5B-D). No change was observed in GST activity between cisplatin and cisplatin + Y27632 treated cells. These data indicate that Y27632 treatment potentially alters the intracellular accumulation of cisplatin as well as enhances DNA repair following cisplatin injury to the DNA. To test the contribution of each of these Y27632 induced cisplatin resistance mechanisms to cell survival, we treated cisplatin or cisplatin + Y27632 treated cells with sham, verapamil (a general ABC-efflux pump inhibitor), or NG123 (a DNA repair inhibitor) and measured cell survival post-treatment. As demonstrated in Fig. 5E, inhibition of cisplatin efflux with verapamil treatment had no effect on Y27632 induced survival following cisplatin treatment; however blocking DNA repair with NG123 reduced Y27632 mediated cell survival to the level of cisplatin treatment only. These data suggest that, in addition to enhanced proliferation rates and altered cell cycle progression, Y27632-mediated survival following cisplatin treatment is dependent on intact DNA damage repair machinery. Moreover, Y-27632 induced drug efflux following cisplatin treatment plays a minor, if any, role in this process.

ROCK inhibition leads to increased NGP neuroblastoma tumor volume. To address the relevance of our in vitro findings, we utilized a CAM tumor model. NGP neuroblastoma cells were pretreated overnight prior to implantation with cisplatin or cisplatin + Y27632, and delivered to the CAM via an implanted gelatin sponge. The growing tumors were supplemented every two days with sham or Y27632. After 5 days, tumors were removed, photographed, and weighed. Y27632 formed larger tumors with a statistically significant increase in weight (Fig. 6). The data suggest that Y27632 treatment leads to rapid recovery and enhanced tumor formation following cisplatin cytoxicity.

\section{Discussion}

In this study, we demonstrate that inhibition of Rho/ROCK signaling using the pharmacological inhibitor Y27632 in a panel of human neuroblastoma cells leads to enhanced cell survival following cytotoxic insult and increased chemoresistance following cisplatin treatment. Moreover, our data indicate that alterations in the expression of cell cycle progression and key cisplatin resistance genes are responsible for the observed survival advantage of Y27632 treated cells. These findings are the first to examine the role of the Rho/ROCK signaling pathway in the regulation of cell survival following chemotherapy, and have direct implications for cancer therapy considering the growing interest in targeting Rho-GTPase signaling as a potential chemotherapeutic treatment of a number of tumor types.

While Rho-GTPases have been suggested as prognostic markers in some tumors and their activity strongly contributes to the metastatic behavior of cancers (22), our current understanding of the contribution of the Rho/ROCK signaling pathway to cell survival/apoptosis is abysmal at best. This pathway has been shown to play both pro-apoptotic and 
pro-survival roles in a number of normal and tumor cells, with few consistent underlying mechanisms explaining this confounding dual role to date. For example, Rho/ROCK signaling has been shown to promote cell survival in melanoma $(10,23)$, gastric (24), and colorectal cancers (25). In contrast, Rho/ROCK signaling promotes apoptosis in prostate cancer $(26,27)$, gastric cancer (28), chronic myelogenous leukemia (29), and lymphoma (30). Moreover, membrane blebbing and nuclear disintegration during apoptosis is dependent on ROCKmediated regulation of the actin myosin cytoskeleton $(31,32)$. Some laboratories have written these obvious discrepancies off as being tissue and cell type dependent, however even within a category of cells such as neurons, obvious contradictions are reported with Rho/ROCK signaling being pro-survival (33-36) or pro-apoptotic (37-43) depending on the reporting group.

Numerous studies have indicated that ROCK activity is essential for programmed cell death. For instance, ROCK activation during apoptosis, in part via caspase-mediated cleavage of ROCK proteins, is essential for apoptotic membrane blebbing, chromosome condensation, Golgi body fragmentation, and eventual phagocytosis via immune cells (13-15,32,44-46). Moreover, ROCK activity promotes a feedback loop which perpetuates caspase- 3 activation and leads to enhancement of apoptosis (27). ROCK has also been shown to initiate apoptosis via PTEN activation (16), a p53-independent induction of p21 expression (26), JNK activation (29), and via Fas/FasL activation $(23,27,47,48)$. While numerous studies have indicated that ROCK serves a pro-apoptotic function, only a handful of reports have shed light on the anti-apoptotic role of ROCK. ROCK signaling reportedly upregulates anti-apoptotic family members and inhibits caspase activation $(25,49)$. Moreover, ROCK promotes cadherin-mediated cell survival through activation of protein kinase D (PKD) and nuclear factor kappa-B (NFKB) (50). Our data suggest that in cisplatin treated neuroblastoma cells, ROCK serves a pro-apoptotic role in part through regulation of cell cycle regulation, p53 signaling, intrinsic and extrinsic apoptotic gene expression, and modulation of cisplatin resistance genes.

While numerous laboratories are designing and utilizing small molecule inhibitors of ROCK for disruption of the metastatic cascade that occurs in malignant tumors, the obvious lack of understanding regarding this signaling pathway's role in the decision between survival and apoptosis should concern both scientists and clinicians. Rho/ROCK inhibition in tumors may very well block tumor cell invasion and metastasis as is strongly reported in the literature (51), but if resistance to anti-tumor therapeutics is markedly increased by this inhibition, then patient survival could be grim even if tumor spread is decreased. We feel the findings of this report may serve as a warning that a better understanding of the multifaceted, complex roles employed by this signaling pathway are needed before implementation of its clinical use as an anti-cancer agent.

\section{Acknowledgements}

We would like to thank Dr Rani George (Harvard Medical School) for the generous use of the neuroblastoma cell lines, Dr Ellen Fynan (Worcester State College) for technical assistance with the tumor CAM assays, and Dr Dianne C.
Mitchell (Acceleron Pharmaceuticals) for theoretical design, technical advice, and manuscript editing. This study was supported by a National Heart, Lung, and Blood Institute Award (HL098931) and a WSC institutional grant awarded to B.A.B.

\section{References}

1. Kamai T, Arai K, Sumi S, et al: The rho/rho-kinase pathway is involved in the progression of testicular germ cell tumour. BJU Int 89: 449-453, 2002.

2. Kamai T, Tsujii T, Arai K, et al: Significant association of Rho/ROCK pathway with invasion and metastasis of bladder cancer. Clin Cancer Res 9: 2632-2641, 2003.

3. Kaneko K, Satoh K, Masamune A, Satoh A and Shimosegawa T: Expression of ROCK-1 in human pancreatic cancer: its downregulation by morpholino oligo antisense can reduce the migration of pancreatic cancer cells in vitro. Pancreas 24: 251-257, 2002.

4. Kleer CG, van Golen KL, Zhang Y, Wu ZF, Rubin MA and Merajver SD: Characterization of RhoC expression in benign and malignant breast disease: a potential new marker for small breast carcinomas with metastatic ability. Am J Pathol 160: 579-584, 2002.

5. Zhou J, Zhao LQ, Xiong MM, et al: Gene expression profiles at different stages of human esophageal squamous cell carcinoma. World J Gastroenterol 9: 9-15, 2003.

6. Croft DR, Sahai E, Mavria G, et al: Conditional ROCK activation in vivo induces tumor cell dissemination and angiogenesis. Cancer Res 64: 8994-9001, 2004.

7. Horiuchi A, Kikuchi N, Osada R, et al: Overexpression of RhoA enhances peritoneal dissemination: RhoA suppression with Lovastatin may be useful for ovarian cancer. Cancer Sci 99: 2532-2539, 2008.

8. Somlyo AV, Bradshaw D, Ramos S, Murphy C, Myers CE and Somlyo AP: Rho-kinase inhibitor retards migration and in vivo dissemination of human prostate cancer cells. Biochem Biophys Res Commun 269: 652-659, 2000.

9. Rattan R, Giri S, Singh AK and Singh I: Rho/ROCK pathway as a target of tumor therapy. J Neurosci Res 83: 243-255, 2006.

10. Routhier A, Astuccio M, Lahey D, et al: Pharmacological inhibition of Rho-kinase signaling with Y-27632 blocks melanoma tumor growth. Oncol Rep 23: 861-867, 2010.

11. Amine A, Rivera S, Opolon P, et al: Novel anti-metastatic action of cidofovir mediated by inhibition of E6/E7, CXCR4 and Rho/ROCK signaling in HPV tumor cells. PLoS One 4: e5018, 2009.

12. Shi J and Wei L: Rho kinase in the regulation of cell death and survival. Arch Immunol Ther Exp (Warsz) 55: 61-75, 2007.

13. Chang J, Xie M, Shah VR, et al: Activation of Rho-associated coiled-coil protein kinase 1 (ROCK-1) by caspase- 3 cleavage plays an essential role in cardiac myocyte apoptosis. Proc Natl Acad Sci USA 103: 14495-14500, 2006.

14. Orlando KA and Pittman RN: Rho kinase regulates phagocytosis, surface expression of GlcNAc, and Golgi fragmentation of apoptotic PC12 cells. Exp Cell Res 312: 3298-3311, 2006.

15. Orlando KA, Stone NL and Pittman RN: Rho kinase regulates fragmentation and phagocytosis of apoptotic cells. Exp Cell Res 312: 5-15, 2006.

16. Kilic E, ElAli A, Kilic U, et al: Role of Nogo-A in neuronal survival in the reperfused ischemic brain. J Cereb Blood Flow Metab 30: 969-984, 2010.

17. Stein GS and Borun TW: The synthesis of acidic chromosomal proteins during the cell cycle of HeLa S-3 cells. I. The accelerated accumulation of acidic residual nuclear protein before the initiation of DNA replication. J Cell Biol 52: 292-307, 1972.

18. Olive PL and Banath JP: The comet assay: a method to measure DNA damage in individual cells. Nat Protoc 1: 23-29, 2006.

19. Mannervik B: The isoenzymes of glutathione transferase. Adv Enzymol Relat Areas Mol Biol 57: 357-417, 1985.

20. Ribatti D, Nico B, Vacca A and Presta M: The gelatin spongechorioallantoic membrane assay. Nat Protoc 1: 85-91, 2006.

21. Stewart DJ: Mechanisms of resistance to cisplatin and carboplatin. Crit Rev Oncol Hematol 63: 12-31, 2007.

22. Chang YW, Bean RR and Jakobi R: Targeting RhoA/Rho kinase and p21-activated kinase signaling to prevent cancer development and progression. Recent Pat Anticancer Drug Discov 4: 110-124, 2009. 
23. Sarrabayrouse G, Synaeve C, Leveque K, Favre G and TilkinMariame AF: Statins stimulate in vitro membrane FasL expression and lymphocyte apoptosis through RhoA/ROCK pathway in murine melanoma cells. Neoplasia 9: 1078-1090, 2007.

24. He Y, Wu GD, Sadahiro T, et al: Interaction of CD44 and hyaluronic acid enhances biliary epithelial proliferation in cholestatic livers. Am J Physiol Gastrointest Liver Physiol 295: G305-G312, 2008.

25. He H, Yim M, Liu KH, Cody SC, Shulkes A and Baldwin GS: Involvement of $\mathrm{G}$ proteins of the Rho family in the regulation of Bcl-2-like protein expression and caspase 3 activation by Gastrins. Cell Signal 20: 83-93, 2008.

26. Xiao L, Eto M and Kazanietz MG: ROCK mediates phorbol ester-induced apoptosis in prostate cancer cells via p21 Cip1 upregulation and JNK. J Biol Chem 284: 29365-29375, 2009.

27. Papadopoulou N, Charalampopoulos I, Alevizopoulos K, Gravanis A and Stournaras C: Rho/ROCK/actin signaling regulates membrane androgen receptor induced apoptosis in prostate cancer cells. Exp Cell Res 314: 3162-3174, 2008.

28. Wang YZ and Feng ZQ: Induction of apoptosis by L-NMMA, via FKHRL1/ROCK pathway in human gastric cancer cells. Biomed Environ Sci 19: 285-291, 2006.

29. Potin S, Bertoglio J and Breard J: Involvement of a Rho-ROCKJNK pathway in arsenic trioxide-induced apoptosis in chronic myelogenous leukemia cells. FEBS Lett 581: 118-124, 2007.

30. Parent N, Sane AT, Droin N and Bertrand R: Procaspase-2S inhibits procaspase- 3 processing and activation, preventing ROCK-1-mediated apoptotic blebbing and body formation in human B lymphoma Namalwa cells. Apoptosis 10: 313-322, 2005.

31. Croft DR, Coleman ML, Li S, et al: Actin-myosin-based contraction is responsible for apoptotic nuclear disintegration. J Cell Biol 168: 245-255, 2005.

32. Coleman ML, Sahai EA, Yeo M, Bosch M, Dewar A and Olson MF: Membrane blebbing during apoptosis results from caspase-mediated activation of ROCK I. Nat Cell Biol 3: 339-345, 2001

33. Garcia-Roman N, Alvarez AM, Toro MJ, Montes A and Lorenzo MJ: Lovastatin induces apoptosis of spontaneously immortalized rat brain neuroblasts: involvement of nonsterol isoprenoid biosynthesis inhibition. Mol Cell Neurosci 17: 329-341, 2001

34. Cerezo-Guisado MI, Alvarez-Barrientos A, Argent R, GarciaMarin LJ, Bragado MJ and Lorenzo MJ: c-Jun N-terminal protein kinase signalling pathway mediates lovastatin-induced rat brain neuroblast apoptosis. Biochim Biophys Acta 1771: 164-176, 2007

35. Focking M, Besselmann $M$ and Trapp T: Statins potentiate caspase-3 activity in immortalized murine neurons. Neurosci Lett 355: 41-44, 2004

36. Kitaoka Y, Kumai T, Lam TT, et al: Involvement of RhoA and possible neuroprotective effect of fasudil, a Rho kinase inhibitor, in NMDA-induced neurotoxicity in the rat retina. Brain Res 1018: 111-118, 2004.
37. Semenova MM, Maki-Hokkonen AM, Cao J, et al: Rho mediates calcium-dependent activation of p38alpha and subsequent excitotoxic cell death. Nat Neurosci 10: 436-443, 2007.

38. Hirose M, Ishizaki T, Watanabe N, et al: Molecular dissection of the Rho-associated protein kinase (p160ROCK)-regulated neurite remodeling in neuroblastoma N1E-115 cells. J Cell Biol 141: 1625-1636, 1998.

39. Lingor P, Tonges L, Pieper N, et al: ROCK inhibition and CNTF interact on intrinsic signalling pathways and differentially regulate survival and regeneration in retinal ganglion cells. Brain 131: 250-263, 2008 .

40. Bowerman M, Beauvais A, Anderson CL and Kothary R: Rhokinase inactivation prolongs survival of an intermediate SMA mouse model. Hum Mol Genet 19: 1468-1478, 2010.

41. Ding J, Yu JZ, Li QY, Wang X, Lu CZ and Xiao BG: Rho kinase inhibitor Fasudil induces neuroprotection and neurogenesis partially through astrocyte-derived G-CSF. Brain Behav Immun 23: 1083-1088, 2009.

42. Tura A, Schuettauf F, Monnier PP, Bartz-Schmidt KU and Henke-Fahle S: Efficacy of Rho-kinase inhibition in promoting cell survival and reducing reactive gliosis in the rodent retina. Invest Ophthalmol Vis Sci 50: 452-461, 2009.

43. Julien S, Schnichels S, Teng H, et al: Purkinje cell survival in organotypic cultures: implication of Rho and its downstream effector ROCK. J Neurosci Res 86: 531-536, 2008.

44. Sebbagh M, Hamelin J, Bertoglio J, Solary E and Breard J: Direct cleavage of ROCK II by granzyme B induces target cell membrane blebbing in a caspase-independent manner. J Exp Med 201: 465-471, 2005.

45. Song Y, Hoang BQ and Chang DD: ROCK-II-induced membrane blebbing and chromatin condensation require actin cytoskeleton. Exp Cell Res 278: 45-52, 2002.

46. Sebbagh M, Renvoize C, Hamelin J, Riche N, Bertoglio J and Breard J: Caspase-3-mediated cleavage of ROCK I induces MLC phosphorylation and apoptotic membrane blebbing. Nat Cell Biol 3: 346-352, 2001.

47. Lai JM, Hsieh CL and Chang ZF: Caspase activation during phorbol ester-induced apoptosis requires ROCK-dependent myosin-mediated contraction. J Cell Sci 116: 3491-3501, 2003.

48. Hebert M, Potin S, Sebbagh M, Bertoglio J, Breard J and Hamelin J: Rho-ROCK-dependent ezrin-radixin-moesin phosphorylation regulates Fas-mediated apoptosis in Jurkat cells. J Immunol 181: 5963-5973, 2008.

49. Yoshida T, Clark MF and Stern PH: The small GTPase RhoA is crucial for MC3T3-E1 osteoblastic cell survival. J Cell Biochem 106: 896-902, 2009.

50. Cowell CF, Yan IK, Eiseler T, Leightner AC, Doppler H and Storz P: Loss of cell-cell contacts induces NF-kappaB via RhoA-mediated activation of protein kinase D1. J Cell Biochem 106: 714-728, 2009.

51. Narumiya S, Tanji M and Ishizaki T: Rho signaling, ROCK and mDia1, in transformation, metastasis and invasion. Cancer Metastasis Rev 28: 65-76, 2009. 